\title{
Describing an IMS by a FNRTPN definition: A VHDL approach
}

\author{
C. A. Graciós Marín', , E. Vargas Soto², A. Díaz Sánchez, \\ ${ }^{1}$ Instituto Tecnológico de Puebla. \\ Av. Tecnológico No. 420, Colonia Maravillas Puebla, Pue. México. 72220 \\ Teléfono (52) 222-229-88-24 Fax (52) 222-222-21-14 \\ ${ }^{2}$ Centro de Ingeniería y Desarrollo Industrial(CIDESI). \\ Av. Pie de la Cuesta \#709 U. Hab. Des. San Pablo,Santiago de Querétaro, Qro. México. \\ Teléfono (52) (443) 2-11-98-00 \\ ${ }^{3}$ Inst. Nal. de Astrofísica, Optica y Electrónica, Luis Enrique Erro No. 1 \\ Tonantzintla, Puebla, Pue. México. P.O. Box 52 Teléfono (52) 222-247-20-11
}

\begin{abstract}
A new Petri net extension and a novel method describing the structure and behaviour of an Intelligent Manufacturing System (IMS), using a VHDL tool, is proposed. The PN extension is defined as Fuzzy Neural Real Time Petri Net (FNRTPN), where the fuzzy part let the intelligent scheduling of the tasks for the IMS, and the neural part calculates the estimation of the parameters of the set point for each resource in the system. At last, VHDL is proposed as a Unified Modelling Language (UML) to express the model of the manufacturing process. The extension and VHDL are used to apply a fuzzy neural control scheme to a FMS.
\end{abstract}

\section{Keywords.}

Intelligent Manufacturing Systems; modelling intelligent process; Simulation; Fuzzy Neural control strategy.

\section{Introduction}

The design, modelling, simulation, fault analysis and control of Flexible Manufacturing Systems, have interested in the last 30 years to academic and industrial communities. Despite several solutions have been proposed, the actual requirements of the manufacturing systems, implying the insertion of intelligence in the elements and devices included in the process [15]. In that way, the active interaction of the human with software and hardware, agility, fault tolerance and the adaptability in general, are decisive characteristics that any intelligent manufacturing system should satisfy [1]. Therefore, the new methods and tools for design, simulation and control, must include a unified modelling language that establish a direct translation between the parameters of the process and the different strategies of intelligent control.

The present work begins with a brief description of the state of the art for modelling, simulation and control of FMS, where Real Time Petri nets are modified to introduce a new extension of PN, and strategies for intelligent control applied in manufacturing systems. After that, fundamental theorems for the new Petri nets extension are expressed. Finally, an example of modelling, simulation and control of an Intelligent Transportation, into an IMS, is described. VHDL will be used for the modelling and control of the IMS, to show the requirements improvement in an actual manufacturing process.

\section{Petri Nets Definitions}

\subsection{Real Time PN model.}

Nowadays, Petri nets have been used as an option for modelling, simulation, analysis and control for manufacturing systems. According to of Venkatesh et al [2], Real Time Petri Nets (RTPN) not only models the manufacturing process, but obtains the direct digital control system, described by a Ladder Logic Diagram (LLD).

A RTPN can be obtained by associating timing, and I/O (input/output) set of information to the untimed $\mathrm{PN}$, and it can be defined as:

$$
\mathrm{RTPN}=\{\mathrm{P}, \mathrm{T}, \mathrm{I}, \mathrm{O}, \mathrm{m}, \mathrm{D}, \mathrm{X}, \mathrm{Y}\}
$$

where:

$\mathrm{P}=\left\{\mathrm{p}_{1}, \mathrm{p}_{2}, \ldots, \mathrm{p}_{\mathrm{m}}\right\}$ is a finite set of places;

$T=\left\{t_{1}, t_{2}, \ldots, t_{n}\right\}$ is a finite set of transitions with $P \cup T=0$ and $\mathrm{P} \cap \mathrm{T}=0$;

I: $\mathrm{P} \times \mathrm{T} \rightarrow \mathrm{N}$ is an input function that defines the set of directed $\operatorname{arcs}$ from $P$ to $T$ where $N=\{1,2, \ldots\}$;

$\mathrm{O}: \mathrm{P} \times \mathrm{T} \rightarrow \mathrm{N}$ is an output function that defines the set of directed arcs from $\mathrm{T}$ to $\mathrm{P}$;

$\mathrm{m}_{\mathrm{i}}: \mathrm{P} \rightarrow \mathrm{N}$, is a marking vector whose $i^{\text {th }}$ component represents the number of tokens in the $i^{\text {th }}$ place. An initial marking vector is denoted by $\mathrm{m}_{0}$;

$\mathrm{D}: \mathrm{T} \rightarrow \mathrm{R}+$, is a firing time function where $\mathrm{R}+$ is the set of non-negative real numbers;

$\mathrm{X}: \mathrm{P} \rightarrow\{-, 0,1,2, \ldots, \mathrm{K}\}$ and $\mathrm{X}(\mathrm{pi}) \neq \mathrm{X}(\mathrm{pj}), \mathrm{i} \neq \mathrm{j}$, is an input signal function, where $\mathrm{K}$ is the maximum number of input signal channels, and " -" is the dummy attribute indicating no assigned channel to the place. (See figure 1).

$\mathrm{Y}: \mathrm{T} \rightarrow \mathrm{L}$, is an output signal function, and $\mathrm{L}$ is a set of integers. 


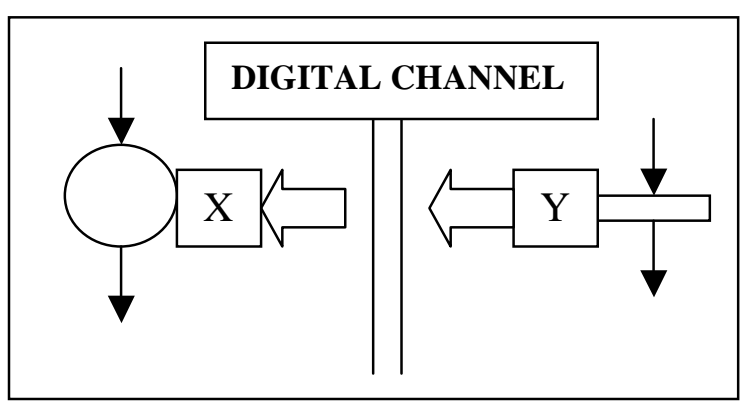

Figure 1. Input and output vectors in RTPN extension

\section{RTPN properties.}

1. Timing vector (D) is intended to associate time delays to transitions modelling the activities in the system.

2. Input signal vector $(\mathrm{X})$ reads the state of the input signals from digital input interface. $\mathrm{X}$ associates attributes to every place. $X_{i}=X\left(p_{i}\right)$, where pi represents the number of channels (bits) of the input pi. The contents of any $X_{i}$ are either 0 or 1 .

3. Output signal vector $(\mathrm{Y})$ is intended to send output signals through digital output interface. $\mathrm{Y}$ associates attribute to every transition. $Y_{i}=Y\left(t_{i}\right)$ is the attribute associated to transition ti which represents the number that is to be sent to the digital output interface.

While the program is executed, RTPN writes the decimal number corresponding to the output channel to digital output interface when a transition is fired.

Using the $\mathrm{X}$ y $\mathrm{Y}$ vectors, a new extension, based on the inclusion of decision strategy by intelligent control, and the parameter acquisition of the process for the regulation the best estimation can be obtained by using a neural network with back propagation training.

In addition, a new variation for hardware description languages, using direct translation for the control expressed in a ladder logic diagram, is available. That new alternative compacts the design and development for the intelligent control in a programmable device, which can be applied at the process modelled [3, 4].

\subsection{The Fuzzy Neural RTPN model}

Several contributions on the application of intelligent control to solve specific problems can found in recent literature. Some propositions, based on intelligent control strategies, allow the timing, sequencing and scheduling of tasks, adding adaptability to process parameter variations, such that velocity, position and used materials $[5,6]$.

Fuzzy logic, neural networks (NN), genetic algorithms and hybrid systems are actually applied in intelligent manufacturing system. In fact, fuzzy and neural networks are the most common structure used to control the activities, like robots, conveyor belts and CNC machines, of specific manufacturing process. Moreover, the inclusion of fuzzy and NN in PN definition to solve the short-term control, are included in recent works [16].

The present work describes a neurofuzzy control scheme, where neural part measures the signal error on the coupled sensors in each one for the regulation of the control parameters, and allows evaluating the task sequencing. Since a fuzzy task scheduler, which determinates the activation of resources according to disposability, can be defined, the goal of intelligent parameter adjusting of each resource of the process, based on previous fuzzy decisions, improves the adaptability.

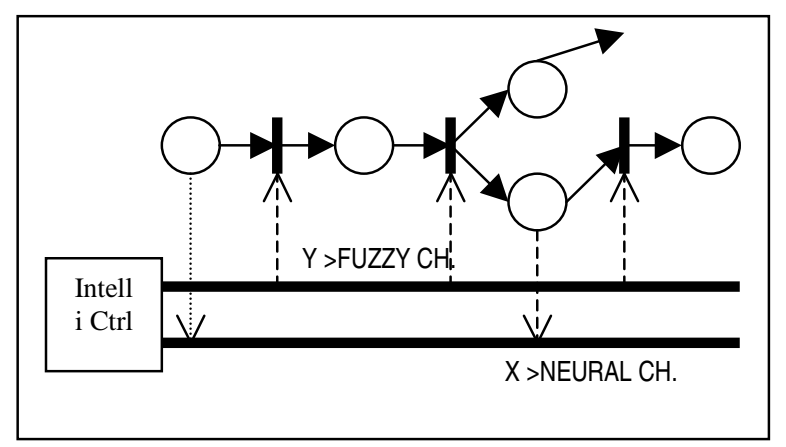

Figure 2. FNRTPN extension

\section{VHDL and Intelligent Control scheme}

Figure 2 shows the FNRTPN extension, where the Y channel add the fuzzy logical decision on the PN firing, which simulates the decision taken to activate the resources of the real process. The NN part is applied to estimate the condition of the resources using a backpropagation scheme. Since the condition of the whole process is known, the availability of the resources and the firing decision of the tasks can be controlled.

In order to include the fuzzy and NN schemes to the original definition of the RTPN, a two mapping function must be firstly defined.

If a new firing vector of the PN F is defined, restricted to the fact that scheduler knows the state of the places in any time of the evolution of the process. The definition of $F$ requires a mapping to translate the fuzzy logical operation values of the scheduler into converted digital values for the original RTPN. That fuzzy scheme provides an intelligent firing procedure for one or more transitions to enable activities (places) of the resources modelled. In other words, $\mathrm{F}$ is indirectly mapped by the firing vector $\mathrm{D}$ to FIRE the change transition of the RTPN actual states. To evaluate the actual state activities, the neural part is connected to the $\mathrm{X}$ digital channel, which recognises the values of the sensors.

Neural networks (NN) can be applied to adjust the parameters for a soft variation in a typical discrete PID control scheme [7]. However, the $\mathrm{X}$ channel is used to modify the condition of the transitions firing (enabling the activities). Since the output of the final neuron convert the real value of the sensor connected in the structure, to a fuzzy value which is evaluated for the scheduler to determine the actual condition of each recourse.

Consequently, the NN identifies the actual values of the resources (position, velocity, pressure, etc) to be known by the scheduler to decide the next activity. Therefore, if $\mathrm{Z}=\left\{\mathrm{z}_{1}, \mathrm{z}_{2}, \ldots, \mathrm{z}_{\mathrm{m}}\right\}$ is the finite set of neurons connected to each $X$ channel, $Z: X \rightarrow P$, where the digital values of the 
sensors are evaluated by the NN to refine the decision of the scheduler and the values to be adjusted in the actuators of each resource, and $\mathrm{m}$ is the number of places, indicating the status of all the resources. Therefore, the number of sensor must be connected to verify the actual condition of each resource.

For the present work, a single-slide back-propagation neural network structure is proposed, because its efficiency in two-slides scheme [7, 12]. The neural scheme is shown in Figure 3. That structure will be only used in the auto tuning strategy for the discrete and analogue actuators of the resources. The final decision on the parameter values will depend on the scheduler.
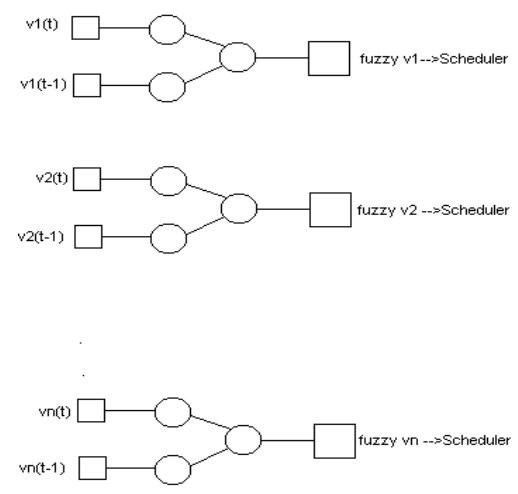

Figure 3. Neural scheme

\subsection{VHDL description for FNRTPN}

VHDL is a suitable tool to describe and model DES by the structural and behavioural definitions in the entity and architecture schemes. Several authors propose VHDL to model structures of intelligent control, incorporating fuzzy, NN, or other kind of schemes applied in FMS for sequencing, scheduling and control.

Using typical command description for discrete systems, a good level definition can be obtained to describe the structure and behaviour of the FNRTPN model.

\subsection{Fuzzy Neural Control Scheme}

The use of a hardware description language is proposed as a tool of simulation and analysis for the FNRTPN model to develop a neurofuzzy control scheme to be applied in the real process. VHDL allows a concurrent design, which uses a unified modelling language with a unique database, generated and addressed at any step of the method.

Despite VHDL is mainly used in the design of complex digital circuits, it can be used in FNRTPN to describe processes like DES. Al last, the simulation, analysis and design of the control can be evaluated using a typical discrete event evolution diagram, where transition signals express the discrete behaviour of the process in terms of the scheduling, timing and sequencing of task.

If the simulation and analysis have been exhaustively realised without find problems in feasibility, the next step for the synthesis of the intelligent control can be made.
Using the report generated for VHDL tool, an Inverse Logistic Algorithm (ILA) can be proposed to obtain the structure of the control scheduler. Therefore, the fuzzy vector $\mathrm{F}$, and the neural set $\mathrm{NN}$ allows the interconnection between the real process and the intelligent control scheme (See figure 2).

The structure and design for the scheduler was proposed by Salapura [8], where the fuzzifier, the defuzzifier, the set of rules and the inference machine are coded in VHDL. Moreover, the behavioural information is obtained by the ILA algorithm applied to the FNRTPN model of the process.

The ILA procedure is as follows:

Each ti (process) is marked as pj (FNCS)

Each pi (process) is marked as tj (FNCS)

$\mathrm{I} \leftarrow \rightarrow \mathrm{O}$ for each state in the structure of the FNRTPN

$\mathrm{A}(\mathrm{FNCS})=\mathrm{A}-1$ (process)

Evolutionary method (Dadone)

ASM description for the structure and behavioural obtained.

Coupling step between the CORE defined for typical resources restricted for CADYC method proposed and the ASM description.

Definition in VHDL.

Testing until complete satisfaction.

That algorithm is based on the scheme suggested by Dadone in 1997 [16], where an evolutionary programming method is presented. The method offers improvements in the use of VHDL, as a unified modelling language, for all the steps required in control schemes if the process is always described like a DES, and the control strategy is implemented in a programmable device.
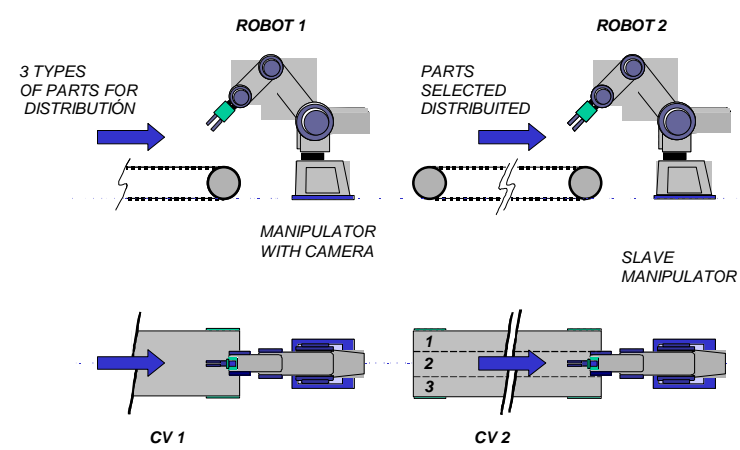

Figure 5. System to be modelled

\section{An Example - Intelligent transport}

Figure 5 shows the layout of the Transport system, which is a part of a FMS analysed in Instituto Tecnológico of Puebla. That system is used for distribution and inspection of the parts manufactured in the FMS. The neurofuzzy control scheme, allows an intelligent adjust for the process. Two Mitsubishi ${ }^{\mathrm{TM}} 5$ degrees of freedom robots are included as resources of manipulation of the pieces. 
The first robot includes a camera vision for the detection of the pieces and the piece can be taken. Two conveyor belts (cv), driven by, servo motors are used for transportation. Additional sensors and actuators are included, as shown at Figure 6.

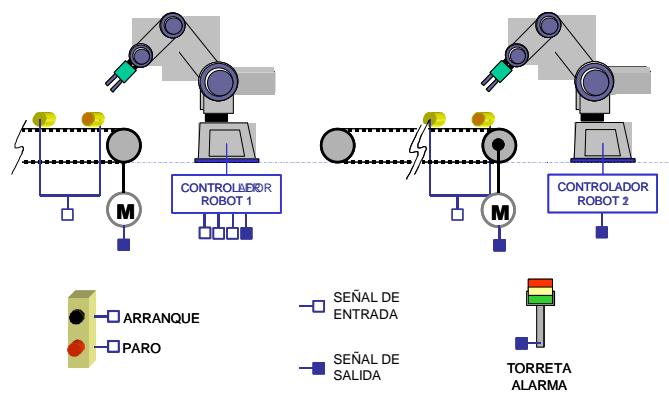

Figura 6. Signals for the system

Figure 6 is an extended layout of the system to be modelled and controlled, where the $\mathrm{X}$ and $\mathrm{Y}$ parameters for the FNRTPN are obtained. By using that information, the structure for the FNRTPN can be created.

Figure 7 shows the the TAD of the transport system. The description is obtained by the application of Woi and Bundell method proposed in [5], and the tool developed in [11]. The ASM description and the FNRTPN model are obtained, and results are shown in Figures 8 y 9.

The obtained ASM description describes the process task sequence and their conditional decisions, where intelligent control can be applied. Figure 9 shows the final FNRTPN model, where the specific tasks of the process are defined and the transitions are finite. Each transition receives a fuzzy channel $f_{i}$ for the scheduler, in order to choose the evolution and adequate sequencing, according to the estimated values of the system by the neural part.

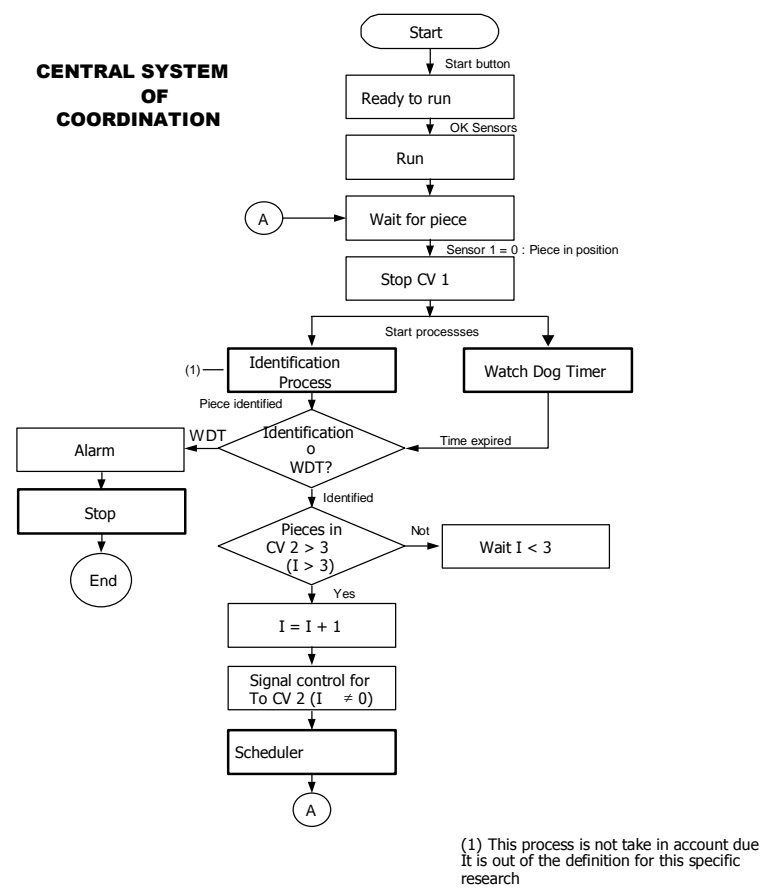

Figure 7. TAD description

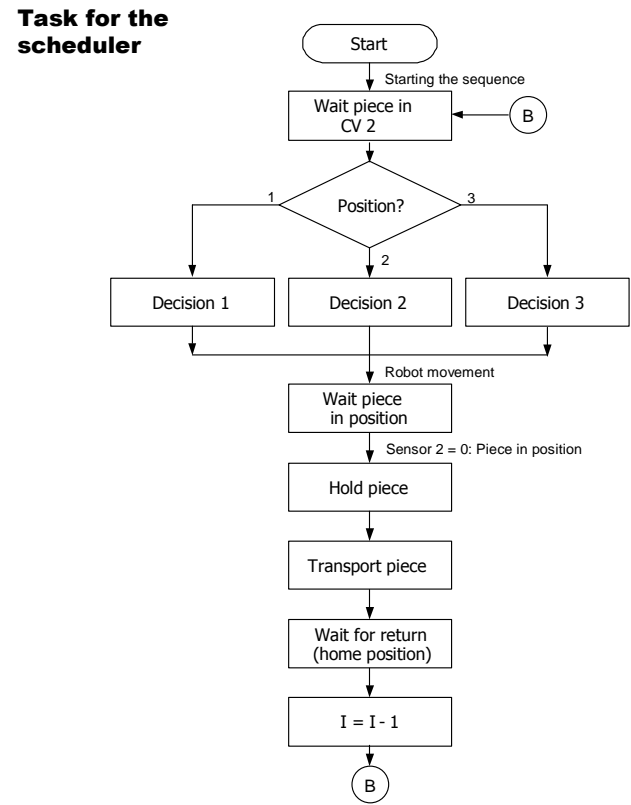

Figure 8. ASM Description

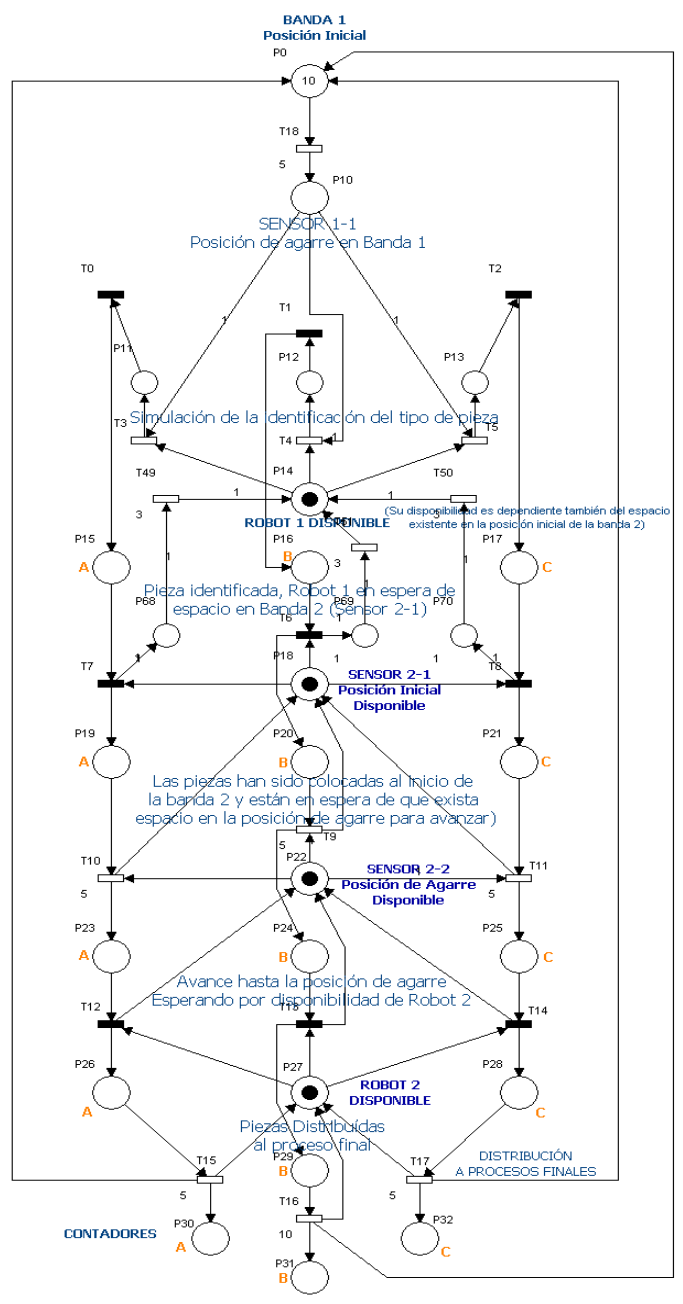

Figure 9. FNRTPN model 
Can be noticed similar transportation tasks for each type of piece, because they have been defined a priori in a VHDL CORE. The discrete model for the servomotors, cv's and robot dynamics, were modelled using linear discrete state variable equations. The student version BASELINE 10.7 of ALTERA $^{\mathrm{TM}}$ was used, and the programming scheme was developed in $\mathrm{C}$ language for MS-DOS ${ }^{\mathrm{TM}}$, using a NETLIST definition. Figure 10 shows the synthetic structural and behavioural description for the FNRTPN model.

The directive entity is used to define the input signals (fuzzy,) and output signals (neural), which are expressed in the PORT command, where all the resources allowed states, and fixed times assigned to specific tasks are given. Figure 11 shows the neurofuzzy control description obtained using the proposed method of ILA applied at the FNRTPN model, where the $\mathrm{X}$ and $\mathrm{Y}$ transposition can be observed and the D's state are determined.

Despite the direct neural control is defined a priori by the CORE, the final decision to change the PID parameters of the actuators resides on the scheduler. Similar values for the Neural PID were pre programmed and obtained as in [12] for the servo motors and the robots. To evaluate the performance of the FNRTPN and the real evolution of the system, a discrete simulation is showed in Figure 12.

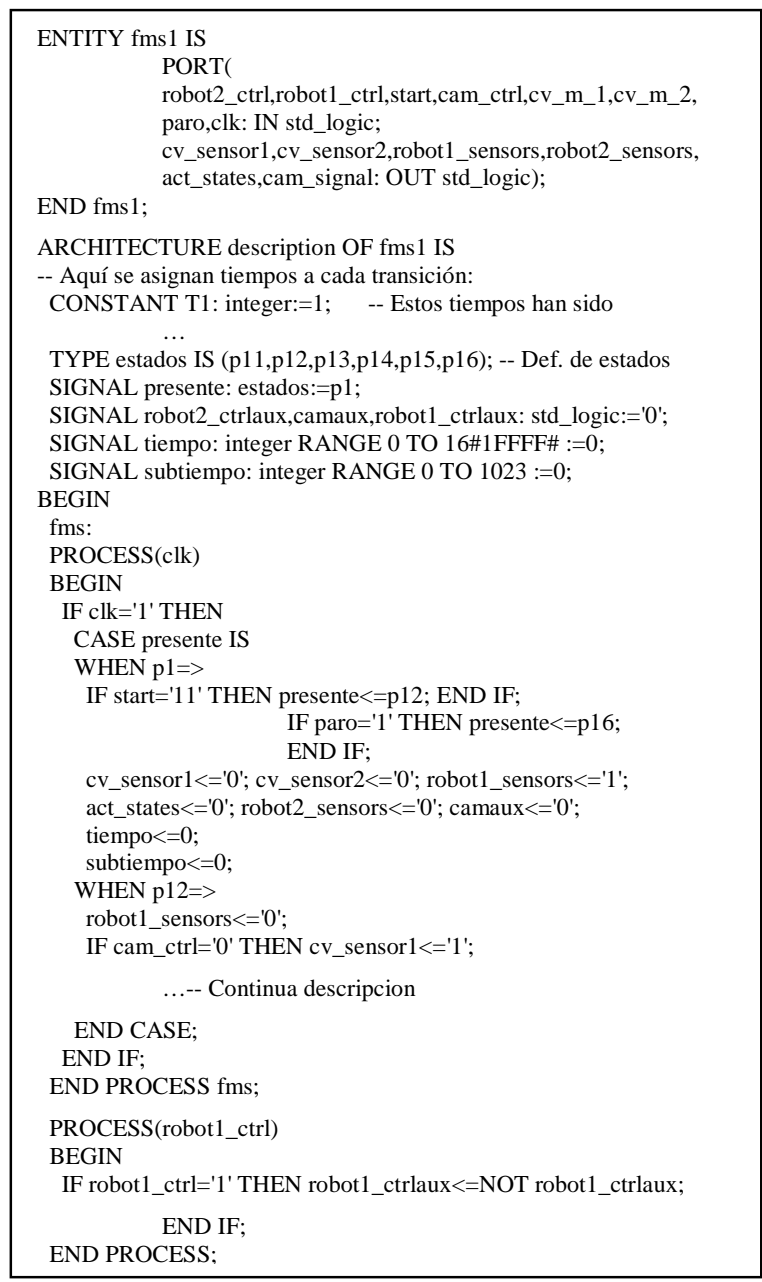

Figure 10. VHDL description of the FNRTPN model.

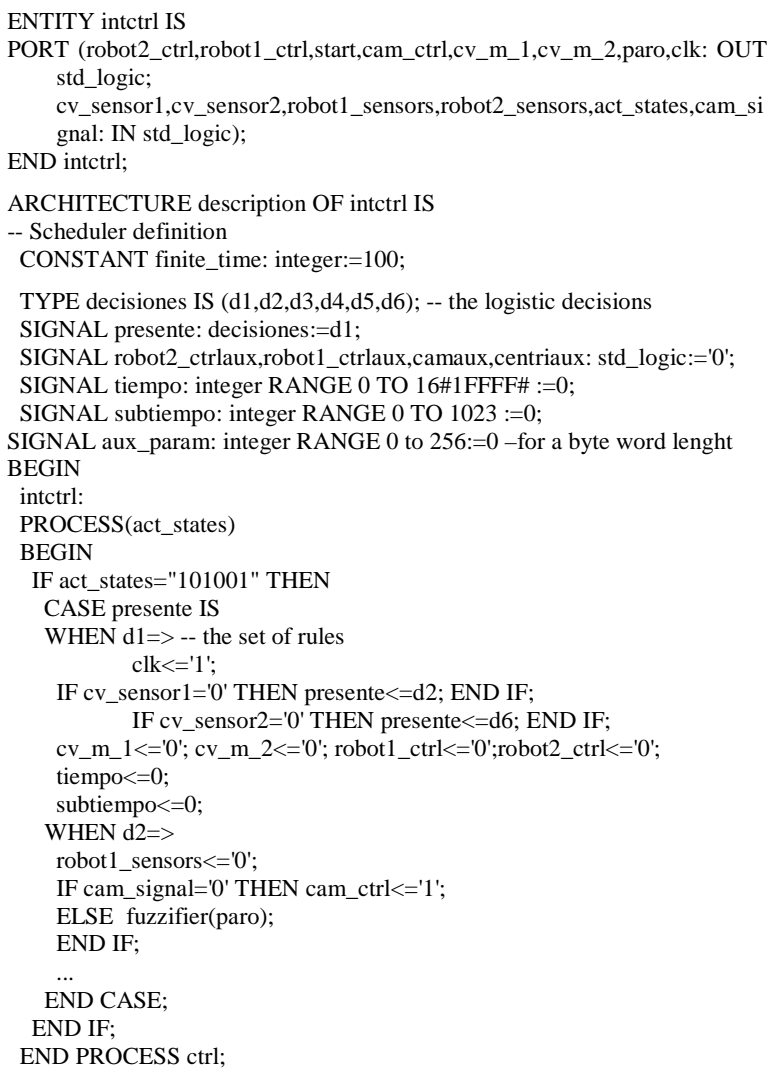

Figure 11. Partial VHDL description of the neurofuzzy control scheme (Part 1)

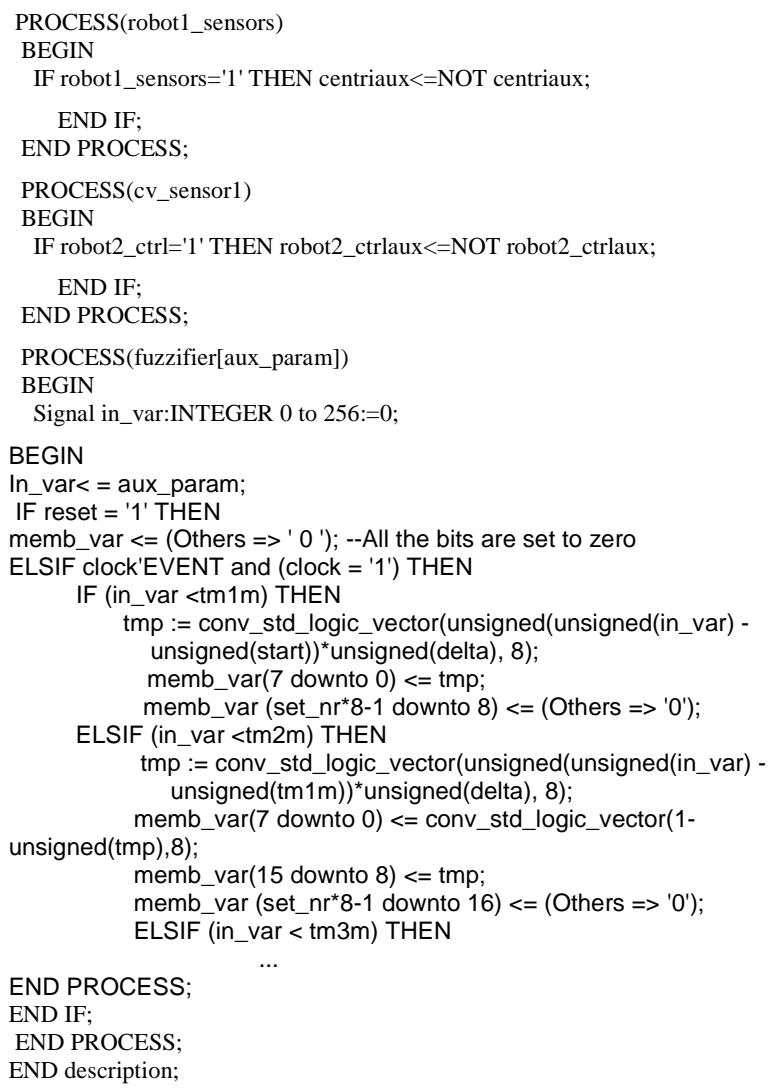

Figure 12. Partial VHDL description of the neurofuzzy control scheme (Part 2) 
The adequate evolution between the real performance of the FMS and the control decisiones of the scheme obtained by our method can be noticed. The evolution of the real system was evaluated in other environments like ARENA $^{\mathrm{TM}}$, OOPN tools, but the observance of the model and the control in the same platform, was hard to realize. Obtained improvements allow the analysis of the discrete dynamics of the process model, and the control decisions can be evaluated. However, some problems related to the resources discrete models were found.

\section{Conclusions and future work.}

The results of the application of the proposed method show improvements in control simplicity, and a better performance when a FMS is modelled, simulated and designed in its control strategy. The intelligent control applied allows to the system self deterministic behaviour, increasing its adjusting and fault tolerance.

Therefore, the method proposed a new extension of PN, with a better link between the real FMS and the intellligent control scheme. The project goals have been fulfilled, and the complete environment is working in the CIM-2000 of the Instituto Tecnológico de Puebla, where the intelligent control system applied shows an improved performance for the programmed activities.

The proposed method demonstrates the feasibility of the application of novel control strategies, which can be restricted by the use of discrete event models of process using discrete state variables for the elements and devices. The robustness and stability of the intelligent control generated must be analysed in future projects.

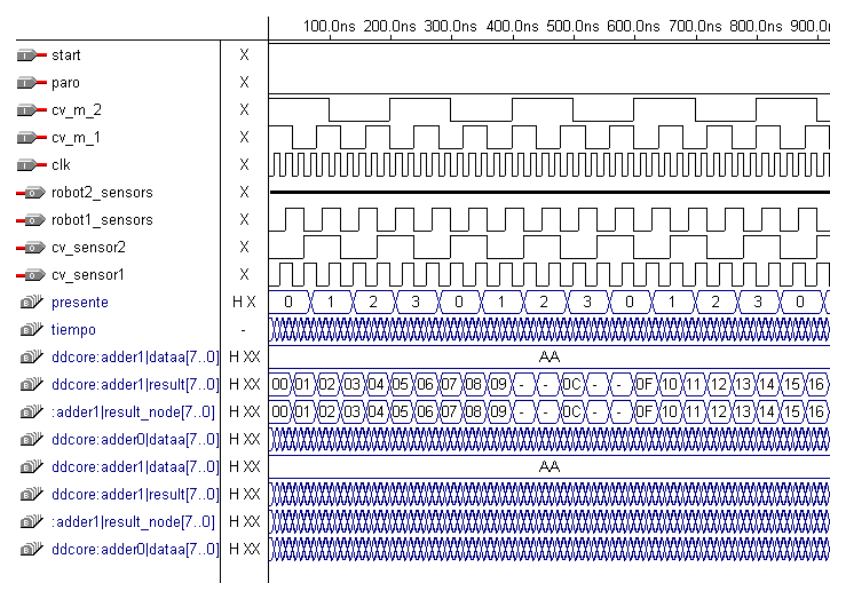

Figure 12. Simulation

\section{Acknowledges.}

The authors want to express thanks to Dr. Dewi Jones and the Instituto Tecnológico of Puebla laboratories, for their valuable help. The present work was financially supported by the Asociación Nacional de Universidades $e$ Instituciones de Educación Superior, the Consejo del
Sistema Nacional de Educación Tecnológica and the Dirección General de Institutos Tecnológicos.

\section{References}

1. Shen W., Norrie D. H., Agent-Based Systems for Intelligent Manufacturing: A State-of-the-Art Survey, Knowledge and Information Systems, an International Journal, 1(2), pp. 129-156, 1999.

2. Gregor E., A Universal Modeling Language, $21^{s t}$ International Conference on Applications and Theory of Petri Nets, Aarthus, Denmark, June 26-30, 2000.

3. Zhou M., Venkatesh, K., Modelling, Simulation and Control of FMS.-A Petri Net approach, World Scientific, 1999.

4. Zimmermann A., Modelling of Manufacturing Systems and Production Routes using Colored Petri Nets, Int. Proc. Of the $3^{\text {rd }}$ IASTED, Int. Conf. On Robotics and Manufacturing, Cancún, México, pp. 380-383, 1995.

5. Woi L. A., Bundel G. A., Analysis of a flexible manufacturing system task controller software model using hierarchical timed Petri Nets, Information systems engineering research group, University of Western Australia, 1999.

6. Van der Aalst, Modelling and Analysis of Production systems using a Petri Net based approach, Eindhoven University of Technology, The Netherlands, 1998.

7. Aguado B. A., Temas de Identificación y Control Adaptable, ICIMAF, La Habana, Cuba, 2000.

8. Salapura V., Hamann V., Implementing Fuzzy Control Systems using VHDL and Statecharts, Technische Universitat Wien, Austria, 2001

9. Gupta P., Hardware-Software codesign, IEEE Potentials, December 2001/2002.

10.Bundell G. A., An fpga Implementation of the Petri net Firing Algorithm, Information Systems Enginnering group, University of Western Australia, 2000.

11.Torres G. H. H., Análisis y modelado de un sistema de manufactura flexible usando redes de Petri Jerárquico Temporizadas, MSc. Thesis, Instituto Tecnológico de Puebla, 2003.

12. C. Gracios, G. Muñoz, J. Estévez and S. Torres, "Neuro-PID control system with gravity compensation," Proc. 3rd IEEE Intl. Symp. on Robotics and Automation, Toluca, Edo. Mex., 2002.

13. Jiang Ch., Zheng Y., Fuzzy Reasoning Based On Petri Nets, IEEE.

14. T. Ojala, "Neuro-Fuzzy Systems for Control," MSc. Thesis, Tampere Technical University, Finland, 1995

15 R. Myong-Gyun and H. Sang-Eun, "Control and Monitoring of Factory Automation System using Fuzzy Petri Nets," IEEE, Int. Symp. on Industrial Electronics, Pusan, Korea, 2001

16. P. Dadone, "Fuzzy Control of Flexible Manufacturing Systems," MSc. Thesis, Blacksburg, VA, 1997.

17. P. Chen and K. Forward, "Fuzzy Petri Nets," $1^{\text {st }}$ Int. Conf. On Knowledge-Based Intelligent Electronic Systems, Adelaide, Australia, 1997 